\title{
Antimicrobial Susceptibility and Genomic Structure of Arcobacter skirrowii Isolates
}

\author{
Ingrid Hänel, Helmut Hotzel, Herbert Tomaso and Anne Busch* \\ Institute of Bacterial Infections and Zoonoses (IBIZ), Friedrich Loeffler Institute, Jena, Germany
}

\section{OPEN ACCESS}

Edited by:

Marcelo Tolmasky, California State University, Fullerton,

United States

Reviewed by: German Matias Traglia, Universidad de Buenos Aires,

Argentina

Greta Gölz,

Freie Universität Berlin, Germany

*Correspondence: Anne Busch anne.busch@fli.de

Specialty section:

This article was submitted to

Antimicrobials, Resistance and Chemotherapy,

a section of the journal

Frontiers in Microbiology

Received: 15 August 2018 Accepted: 28 November 2018 Published: 14 December 2018

Citation:

Hänel I, Hotzel H, Tomaso H and Busch A (2018) Antimicrobial Susceptibility and Genomic Structure

of Arcobacter skirrowii Isolates.

Front. Microbiol. 9:3067.

doi: 10.3389/fmicb.2018.03067
Campylobacter spp. are considered the most common bacterial cause of foodborne gastroenteritis in the world. The family Campylobacteraceae includes the genus Arcobacter with the three species Arcobacter butzleri, Arcobacter cryaerophilus, and Arcobacter skirrowii as emergent enteropathogens and potential zoonotic agents. Here, we characterized genome sequences of Arcobacter that were isolated from water poultry on farms in Germany. Isolates were cultured, identified by MALDI-TOF MS and identification was verified with PCR assays. Antibiotic susceptibility testing of isolates was carried out with erythromycin, ciprofloxacin, doxycycline, tetracycline, gentamicin, and streptomycin using the gradient strip method (E-test). We also sequenced whole genomes and predicted antibiotic resistance determinants, virulence factors, performed a phylogenetic analysis to determine the genetic relatedness of these isolates and searched for plasmids.

Keywords: Arcobacter skirrowii, Arcobacter butzleri, antimicrobial susceptibility, genomic structure, antibiotic, genome, antibiotic susceptibility, animal health

\section{INTRODUCTION}

Campylobacter spp. are considered to be the most common bacterial cause of human gastroenteritis in the world (WHO, 2018). In 1991, the new genus Arcobacter (A.) was introduced within the family of Campylobacteraceae. Three species, Arcobacter butzleri, Arcobacter cryaerophilus, and Arcobacter skirrowii, are considered potential zoonotic agents (Prouzet-Mauleon et al., 2006; Levican et al., 2013; Van den Abeele et al., 2014, 2016). Arcobacter spp. are present in the digestive tract of healthy animals (Ünver et al., 2013), but are also associated with enteritis and reproductive disorders in animals (De Smet et al., 2012). Arcobacter can be transmitted to humans by contaminated food (e.g., poultry products) and water (Collado and Figueras, 2011; Hänel et al., 2016).

Isolation of A. skirrowii is often difficult due to its special growth requirements and data on the antibiotic susceptibility of the bacteria are scarce (Yesilmen et al., 2014). Only few representative genomes (for example NCBI, BioProject: PRJNA307998, BioSample: SAMN04386098) have been described.

The aim of this study was to determine the antimicrobial susceptibility of A. skirrowii isolated from domestic water poultry to six antibiotics commonly used to treat diarrhea in humans. The genomic features of A. skirrowii isolates were analyzed to improve diagnostic and antibiotic treatment options. 


\section{MATERIALS AND METHODS}

Arcobacter isolates were cultivated from fecal samples collected in water poultry farms in Thuringia, Germany. A two-step enrichment procedure was done in Arcobacter broth (Oxoid, Wesel, Germany) supplemented with antibiotics (cefoperazone, amphotericin and teicoplanin; CAT, Oxoid) under microaerobic conditions $\left(5 \% \mathrm{O}_{2}, 10 \% \mathrm{CO}_{2}\right.$, and $\left.85 \% \mathrm{~N}_{2}\right)$ for $48 \mathrm{~h}$ at $30^{\circ} \mathrm{C}$. Subsequently, the broth was streaked on plates (MuellerHinton/CAT/5\% defibrinated bovine blood) and incubated under microaerobic conditions for another $24-48 \mathrm{~h}$ at $30^{\circ} \mathrm{C}$. Suspicious colonies were recultivated and identified by matrix-assisted laser desorption/ionization time-of-flight mass spectrometry (MALDI-TOF MS) as described before (El-Ashker et al., 2015; Busch et al., 2018). IVD Bacterial Test Standard, Biotyper 3.1 software, and the database DB 4613 (all Bruker Daltonik GmbH, Bremen, Germany) containing spectra of all Arcobacter species were used. A confirmation of the species identification was performed using a multiplex PCR assay (Douidah et al., 2014).

Antimicrobial susceptibility to six antibiotics (erythromycin, ciprofloxacin, doxycycline, tetracycline, gentamicin, and streptomycin) was determined using the gradient strip diffusion method (E-test ${ }^{\mathrm{TM}}$, bioMérieux, Nürtingen, Germany) following the manufacturer's instructions (Table 1). The bacterial suspensions for the E-test were adjusted to an optical density of 0.1 at $600 \mathrm{~nm}$ (corresponding to approximately 3 to $5 \times 10^{8} \mathrm{cfu} / \mathrm{ml}$ ) in PBS. $750 \mu \mathrm{l}$ were evenly spread on a $\mathrm{MH}$ agar plate and a single strip was put on each plate. After $48 \mathrm{~h}$ of incubation at $30^{\circ} \mathrm{C}$ under microaerobic conditions, the minimum inhibitory concentration (MIC) was determined. The type strain of A. skirrowii DSM 7302 was used as control. For erythromycin, ciprofloxacin, doxycycline, and tetracycline interpretative criteria were based upon EUCAST breakpoints for Campylobacter. For gentamicin EUCAST Enterobacteriaceae breakpoints were applied. For streptomycin the cut-off value for Campylobacter jejuni was used as suggested by the EFSAWorking Group (European Food Safety Authority [EFSA], 2008). The phenotypes were classified as sensitive (S), resistant (R), or intermediate (I) (Table 1).

DNA for whole genome sequencing (WGS) was prepared from colonies harvested from plates. DNA was purified (High Pure PCR Template Preparation Kit; Roche Diagnostics, Mannheim, Germany) and sequencing libraries were generated using the Nextera XT DNA Library Prep Kit (Illumina, Inc., San Diego, CA, United States). From an Illumina MiSeq run 111,0008,300,000 paired-end reads were generated (mean sequencing depth: 29-216 reads). The assignment of the taxonomic labels to all reads was performed with MetaPhlAn (Segata et al., 2012) and Kraken version 0.10.6 (Wood and Salzberg, 2014). Further read processing included quality trimming and assembly with SPAdes 3.9.1 (-careful) (Bankevich et al., 2012) and filtering by removing contigs with a coverage $<5$ and a length $<500$. Quality was assessed with QUAST 4.3 (Gurevich et al., 2013). Annotation was performed with Prokka using the recommended standard settings (Seemann, 2014). Additionally, PhyloPhlAn was used to assign microbial phylogeny (Segata et al., 2013) and visualized with Dendroscope (Huson et al., 2007; Figure 1).
Subsystem category distribution of the assemblies was done with RAST and SEED (Supplementary Figure 1; Aziz et al., 2008; Brettin et al., 2015). Based on ARIBA in the standard settings (Hunt et al., 2017) several databases were used to identify single nucleotide polymorphisms directly from short reads. Resistance genes were predicted using the ResFinder (Zankari et al., 2012) and the PlasmidFinder was used for the analysis of plasmids (Carattoli et al., 2014). Multilocus Sequence Typing (MLST) was done using the MLST database (Carattoli et al., 2014) and for detection of virulence factors VFDB_full was used (Chen et al., 2005). No reads mapped to reference genes. Therefore, no local assemblies were run. Virulence-associated genes known from the literature, i.e., the genes ciaB (HF935951), cj1349 (HF935963), and $c a d F$ (HF935942) were mapped with Geneious (Kearse et al., 2012) on all assemblies and to the known A. skirrowii sequence LRUX01000036.1. Further search for plasmids was done with Bandage (Wick et al., 2015).

All isolates were submitted to the Leibniz Institute, DSMZGerman Collection of Microorganisms and Cell Cultures ${ }^{1}$, and are available under the following DSM numbers: DSM 107942 (A. butzleri FLI 17-1168), DSM 107960 (A. skirrowii FLI 171208-1), DSM 107961 (A. skirrowii FLI 17-1201-3), DSM 107962 (A. skirrowii FLI 17-1208-2), DSM 107963 (A. skirrowii FLI 17-1201-4), DSM 107964 (A. skirrowii FLI 17-1206-2). Wholegenome sequences, assemblies, and raw data of all isolates were submitted under the BioProject PRJNA464281.

\section{RESULTS}

Five A. skirrowii and one A. butzleri isolates were cultivated and identified by MALDI-TOF MS, and due to scores $<2,3$ confirmed by a multiplex PCR assay. As an example, the MALDITOF MS mass list of a wildtyp A. skirrowii 1208_2 is given in Supplementary Table 4. Reference spectra were generated for A. skirrowii wild type isolates (spectra are available upon request).

Taxonomic analysis of the WGS data with MetaPhlAn and Kraken resulted mostly in "unclassified Arcobacter" for A. skirrowii isolates, whereas A. butzleri could be assigned to the correct species. Also analyses based on the genomic features by sequence identity with RAST showed only a relationship to A. butzleri RM4018 for all isolates. A correct taxonomic differentiation of the species was possible with PhyloPhlAn.

The antibiotic susceptibility test results for the Arcobacter isolates obtained by E-test are shown in Table 1. All A. skirrowii isolates were susceptible to erythromycin, ciprofloxacin, doxycycline, tetracycline, and gentamicin and showed resistance to streptomycin. The A. butzleri isolate was susceptible to ciprofloxacin and doxycycline, showed intermediate resistance to gentamicin and was resistant to erythromycin, tetracycline, and streptomycin. Based on the WGS data no resistance determinants were predicted with the ResFinder.

The mean coverage obtained for the isolates using WGS was $>170$ except for A. skirrowii isolate 17-1201-3 with a mean coverage of only 20 . All sequences were assembled and annotated

\footnotetext{
${ }^{1}$ https://www.dsmz.de
} 
TABLE 1 | Antibiotic susceptibility of Arcobacter spp.

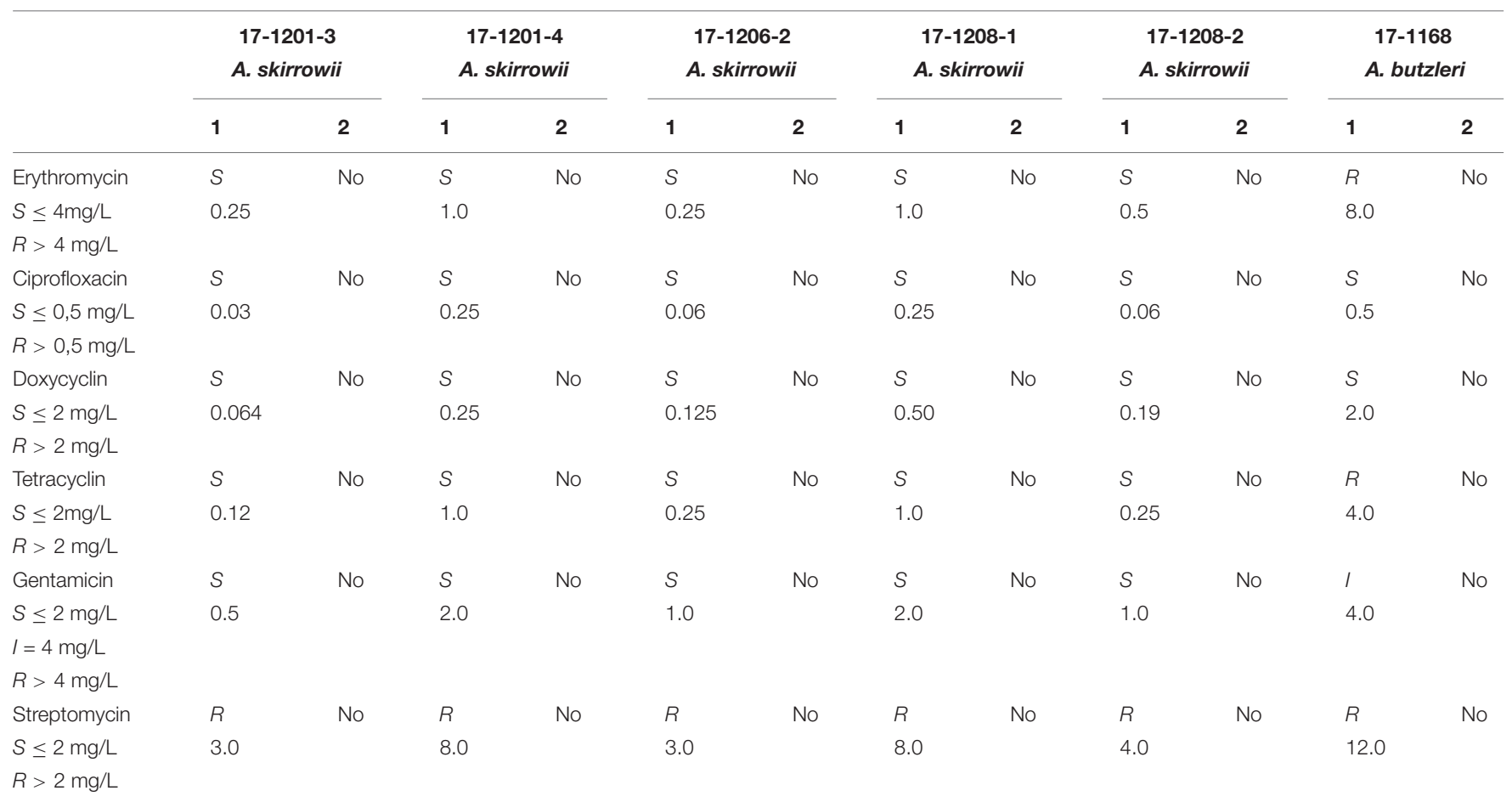

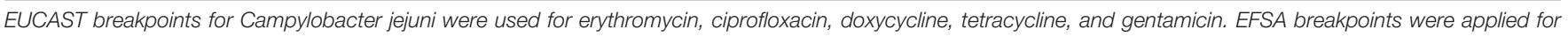
streptomycin (European Food Safety Authority [EFSA], 2008). S, susceptible; R, resistant; I, intermediate; 1, laboratory results; 2, ResFinder prediction.

(Supplementary Tables 1, 2). Assemblies consisted of 36 to 145 contigs. Sequence length was predicted to be between $1,911,841$ and $1,940,887$ concordant bases. Due to the lower coverage of A. skirrowii isolate 17-1201-3 the assembly resulted in more contigs, but genome sizes of all A. skirrowii were estimated to be around 1.9 million bases with approximately 1,980 coding sequences. The A. butzleri isolate 17-1168 was estimated to have a genome size of 2.2 million bases and included more coding sequences $(2,143)$ than the $A$. skirrowii isolates. The GC content was determined to be $27.6 \%$ for A. skirrowii and $26.9 \%$ for $A$. butzleri, respectively. When the programs were applied with standard settings, no reads mapped to reference genes in the database, therefore no local assemblies were run. However, the known virulence-associated genes (ciaB (HF935951), cj1349 (HF935963), hecA (HF935064), and cadF (HF935942)) could be mapped to all assemblies and the published $A$. skirrowii sequence LRUX01000036.1. However, all genes mapped with a low sequence identity (49.9 to $87 \%)$. MLST results were extracted from the WGS data and were assigned to new sequence types (ST) (Supplementary Table 3), which were published within the PubMLST.org (ID 888-892). Verification of the results by traditional sequencing methods for MLST was not done.

The analysis of subsystem category distribution showed that the carbohydrate metabolism genes were comparable between A. skirrowii and A. butzleri. For A. skirrowii less elements could be classified for phages, prophages, transposable elements, plasmids, and a reduced number of genes related to potassium metabolism, iron metabolism, and iron acquisition.

\section{DISCUSSION}

The relevance of Arcobacter as a pathogen for humans has not yet been clarified, although three species including A. butzleri, A. cryaerophilus, and A. skirrowii have been associated with gastrointestinal diseases (Collado and Figueras, 2011). It can be assumed that the importance of Arcobacter in human infections is underestimated. For the isolation of $A$. skirrowii special cultivation procedures and therefore adequate detection and identification methods are not available in many laboratories. The taxonomic analysis of WGS data based on reads (MetaPhlAn and Kraken) also relies on yet incomplete databases, so most bioinformatics methods will not detect $A$. skirrowii. The taxonomic assignment based on assemblies and annotation with PhyloPhlAn proved as a fast and efficient method. PhyloPhlAn is a method using $>400$ proteins optimized from among 3,737 genomes thus reflecting more functional differences than phylogenies based on nucleotides.

Data on antimicrobial susceptibility of A. butzleri or A. cryaerophilus are scarce and almost lacking for A. skirrowii isolates. A. skirrowii seems to be susceptible to many antimicrobials (Houf et al., 2001). Although microdilution assays are usually favored, antibiotic testing is not standardized yet and the gradient strip method seems to be a more robust approach for these bacteria (Van den Abeele et al., 2014).

Antibiotic resistance can be induced by gene transfer or plasmid transfer events for example between human, animal, and plant-associated bacteria with streptomycin (Sundin and Bender, 1996). A. butzleri or A. cryaerophilus isolates 


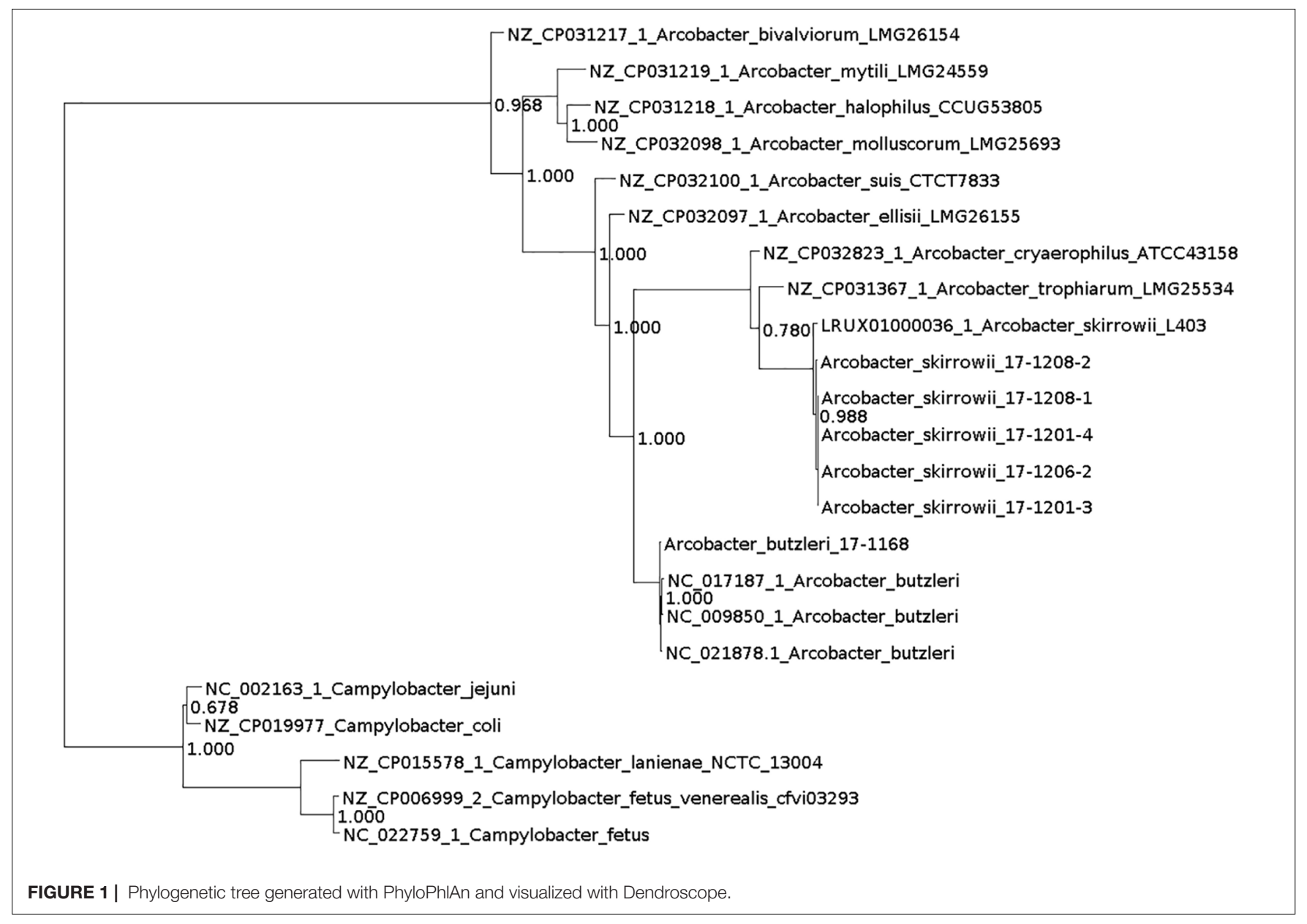

from Europe (Van den Abeele et al., 2016) showed susceptibility to gentamicin, tetracycline, erythromycin, ciprofloxacin, and doxycycline and sometimes resistance to ampicillin. The here studied A. skirrowii isolates were more sensitive and showed only resistance to streptomycin. The CDC reported resistance to ciprofloxacin for almost $25 \%$ of human Campylobacter isolates and $2 \%$ were resistant to azithromycin (CDC, 2015). The antibiotic resistance of the $A$. butzleri isolate could not be predicted with the applied bioinformatics tools.

Antibiotic resistance is often mediated by plasmids especially in the Enterobacteriaceae family (Nikaido, 2009). Plasmids are commonly present in diverse prokaryotes, play an important role in the genetic evolution and adaptation of bacteria and were reported in $9.9 \%$ of $A$. butzleri isolates (Harrass et al., 1998; Toh et al., 2011). Plasmids could be neither predicted by the PlasmidFinder nor by visual inspection of the graphical assembly graphs (Wick et al., 2015) for any of the isolates. In agarose gels no plasmids could be detected comparable with Becker et al. (2016).

Multilocus Sequence Typing is a traditional technique that characterizes isolates using DNA sequences of multiple housekeeping genes. All isolates were assigned to distinct alleles and could be defined as new ST (Supplementary Table 3). Although no virulence factors could be predicted for these
Arcobacter isolates, four genes known to contribute to host adherence and invasion could be mapped with low sequence identity (Levican et al., 2013). The low sequence identity was also an indication that $A$. skirrowii might show more variability within the nucleotide sequences which was not reflected in the databases, yet.

\section{CONCLUSION}

In conclusion, A. skirrowii is a known, but rarely detected pathogen. The main reasons might be slow growth on culture media, overgrowth by other bacteria and underrepresentation in databases. Procedures in most routine microbiology laboratories need to be adapted for the detection and identification of this pathogen. Antibiotic susceptibility testing of A. skirrowii is preferably done using the gradient strip method due to the fastidious growth of the bacteria. Prediction of antibiotic susceptibility based on WGS data should be treated with caution.

Resistance to erythromycin, tetracycline, and streptomycin was found only in A. butzleri, while A. skirrowii was only resistant to streptomycin. Macrolides (here represented by erythromycin) are the preferred therapeutic agents in Campylobacter infections, but they are not necessarily first-choice antibiotics for Arcobacter 
infections for which tetracycline was proposed for severe cases only (Yan et al., 2000; Kayman et al., 2012; Arguello et al., 2015). An important factor in the development of resistance to antimicrobial agents is the uncontrolled use of antibiotics in animal husbandry. Monitoring and reporting of antimicrobial resistance data as well as WGS data analysis of Campylobacter and Arcobacter from domestic animals are important to monitor the evolution of antimicrobial resistance and to optimize diagnostics.

Here, solutions for diagnostic problems working with A. skirrowii were evaluated. Cultivation protocols were provided, MALDI-TOF MS spectra were made available and sequencing data were published in the NCBI, so that the software tools based on the RefSeq (such as BLAST and Kraken) will allow quick identification. The usage of open source software allows an economic and transparent application of the here established analysis. The investigated isolates were deposited in the open collection of the DSMZ. Our data can improve the diagnostic capabilities also of other laboratories and contribute to future work on epidemiological, pathogenetic, and functional analysis of these rarely recognized bacteria. This may further help to elucidate the mechanisms underlying the pathogenicity of A. skirrowii. The comprehensive bioinformatics analysis allows optimizing database dependent bioinformatics tools (for example the ResFinder, PubMLST, or Kraken). The analysis of the function of antibiotic resistance of new developing resistances is important to be able to compare the genetic makeup of resistant and susceptible strains. It can be speculated that the efforts undertaken to eradicate Campylobacter spp. (as cause of gastroenteritis) from the microbiome of farmed animals will lead to a replacement by Arcobacter spp. Then an assessment of the pathogenicity of Arcobacter spp. will be crucial.

\section{REFERENCES}

Arguello, E., Otto, C. C., Mead, P., and Babady, N. E. (2015). Bacteremia caused by Arcobacter butzleri in an immunocompromised host. J. Clin. Microbiol. 53, 1448-1451. doi: 10.1128/JCM.03450- 14

Aziz, R. K., Bartels, D., Best, A. A., Dejongh, M., Disz, T., Edwards, R. A., et al. (2008). The RAST server: rapid annotations using subsystems technology. BMC Genomics 9:75. doi: 10.1186/1471-2164-9-75

Bankevich, A., Nurk, S., Antipov, D., Gurevich, A. A., Dvorkin, M., Kulikov, A. S., et al. (2012). SPAdes: a new genome assembly algorithm and its applications to single-cell sequencing. J. Comput. Biol. 19, 455-477. doi: 10.1089/cmb.2012. 0021

Becker, L., Steglich, M., Fuchs, S., Werner, G., and Nubel, U. (2016). Comparison of six commercial kits to extract bacterial chromosome and plasmid DNA for MiSeq sequencing. Sci. Rep. 6:28063. doi: 10.1038/srep 28063

Brettin, T., Davis, J. J., Disz, T., Edwards, R. A., Gerdes, S., Olsen, G. J., et al. (2015). RASTtk: a modular and extensible implementation of the RAST algorithm for building custom annotation pipelines and annotating batches of genomes. Sci. Rep. 5:8365. doi: 10.1038/srep08365

Busch, A., Thomas, P., Zuchantke, E., Brendebach, H., Neubert, K., Gruetzke, J., et al. (2018). Revisiting Francisella tularensis subsp. holarctica, causative agent of tularemia in germany with bioinformatics: new insights in genome structure, DNA methylation and comparative phylogenetic analysis. Front. Microbiol. 9:344. doi: 10.3389/fmicb.2018.00344

Carattoli, A., Zankari, E., Garcia-Fernandez, A., Voldby Larsen, M., Lund, O., Villa, L., et al. (2014). In silico detection and typing of plasmids using

\section{AUTHOR CONTRIBUTIONS}

$\mathrm{IH}, \mathrm{HH}, \mathrm{AB}$, and $\mathrm{HT}$ have jointly conceived the study. IH provided strains, strain information, and metadata and antibiotic testing to the samples. $\mathrm{AB}$ performed bioinformatics analysis of genomes, assembly, and phylogenetic relationship.

\section{FUNDING}

For work IH was participating in a project of the FriedrichLoeffler-Institut (Antimicrobial Resistance - Research Network T3; HJ-002) and $\mathrm{AB}$ was supported by a grant of the German Federal Ministry of Education and Research within the framework of the project Ess-B.A.R. (FKZ 13N13983). The funders had no role in study design, data collection, and interpretation, or the decision to submit the work for publication.

\section{ACKNOWLEDGMENTS}

We thank Sandra Thierbach, Johannes Solle, Byrgit Hofmann, Peggy Methner, and Anja Hackbart for skillful technical assistance. Finally, yet importantly, we are immensely grateful to Dr. Christine Ahlers for providing samples.

\section{SUPPLEMENTARY MATERIAL}

The Supplementary Material for this article can be found online at: https://www.frontiersin.org/articles/10.3389/fmicb. 2018.03067/full\#supplementary-material

PlasmidFinder and plasmid multilocus sequence typing. Antimicrob. Agents Chemother. 58, 3895-3903. doi: 10.1128/AAC.02412-14

CDC (2015). National Antimicrobial Resistance Monitoring System for Enteric Bacteria (NARMS): Human Isolates Final Report, 2013. Atlanta, GA: US Department of Health and Human Services.

Chen, L., Yang, J., Yu, J., Yao, Z., Sun, L., Shen, Y., et al. (2005). VFDB: a reference database for bacterial virulence factors. Nucleic Acids Res. 33, D325-D328.

Collado, L., and Figueras, M. J. (2011). Taxonomy, epidemiology, and clinical relevance of the genus Arcobacter. Clin. Microbiol. Rev. 24, 174-192. doi: 10. 1128/CMR.00034-10

De Smet, S., De Zutter, L., and Houf, K. (2012). Spatial distribution of the emerging foodborne pathogen Arcobacter in the gastrointestinal tract of pigs. Foodborne Pathog. Dis. 9, 1097-1103. doi: 10.1089/fpd.2012.1184

Douidah, L., De Zutter, L., Van Nieuwerburgh, F., Deforce, D., Ingmer, H., Vandenberg, O., et al. (2014). Presence and analysis of plasmids in human and animal associated Arcobacter species. PLoS One 9:e85487. doi: 10.1371/journal. pone. 0085487

El-Ashker, M., Gwida, M., Tomaso, H., Monecke, S., Ehricht, R., El-Gohary, F., et al. (2015). Staphylococci in cattle and buffaloes with mastitis in Dakahlia Governorate, Egypt. J. Dairy Sci. 98, 7450-7459. doi: 10.3168/jds.2015-9432

European Food Safety Authority [EFSA] (2008). Harmonised monitoring of antimicrobial resistance in Salmonella and Campylobacter isolates from food animals in the European Union. Clin. Microbiol. Infect. 14, 522-533. doi: 10. 1111/j.1469-0691.2008.02000.x

Gurevich, A., Saveliev, V., Vyahhi, N., and Tesler, G. (2013). QUAST: quality assessment tool for genome assemblies. Bioinformatics 29, 1072-1075. doi: 10 . 1093/bioinformatics/btt086 
Hänel, I., Tomaso, H., and Neubauer, H. (2016). Arcobacter - an underestimated zoonotic pathogen? Bundesgesundheitsblatt Gesundheitsforschung Gesundheitsschutz 59, 789-794.

Harrass, B., Schwarz, S., and Wenzel, S. (1998). Identification and characterization of Arcobacter isolates from broilers by biochemical tests, antimicrobial resistance patterns and plasmid analysis. Zentralbl. Veterinarmed. B 45, 87-94.

Houf, K., Devriese, L. A., De Zutter, L., Van Hoof, J., and Vandamme, P. (2001). Susceptibility of Arcobacter butzleri, Arcobacter cryaerophilus, and Arcobacter skirrowii to antimicrobial agents used in selective media. J. Clin. Microbiol. 39, 1654-1656. doi: 10.1128/JCM.39.4.1654-1656.2001

Hunt, M., Mather, A. E., Sanchez-Buso, L., Page, A. J., Parkhill, J., Keane, J. A., et al. (2017). ARIBA: rapid antimicrobial resistance genotyping directly from sequencing reads. Microb. Genom. 3:e000131. doi: 10.1099/mgen.0.000131

Huson, D. H., Richter, D. C., Rausch, C., Dezulian, T., Franz, M., and Rupp, R. (2007). Dendroscope: an interactive viewer for large phylogenetic trees. BMC Bioinformatics 8:460. doi: 10.1186/1471-2105-8-460

Kayman, T., Abay, S., Hizlisoy, H., Atabay, H. I., Diker, K. S., and Aydin, F. (2012). Emerging pathogen Arcobacter spp. in acute gastroenteritis: molecular identification, antibiotic susceptibilities and genotyping of the isolated arcobacters. J. Med. Microbiol. 61, 1439-1444. doi: 10.1099/jmm.0. 044594-0

Kearse, M., Moir, R., Wilson, A., Stones-Havas, S., Cheung, M., Sturrock, S., et al. (2012). Geneious basic: an integrated and extendable desktop software platform for the organization and analysis of sequence data. Bioinformatics 28, 1647-1649. doi: 10.1093/bioinformatics/bts199

Levican, A., Alkeskas, A., Gunter, C., Forsythe, S. J., and Figueras, M. J. (2013). Adherence to and invasion of human intestinal cells by Arcobacter species and their virulence genotypes. Appl. Environ. Microbiol. 79, 4951-4957. doi: 10.1128/AEM.01073-13

Nikaido, H. (2009). Multidrug resistance in bacteria. Annu. Rev. Biochem. 78, 119-146. doi: 10.1146/annurev.biochem.78.082907.145923

Prouzet-Mauleon, V., Labadi, L., Bouges, N., Menard, A., and Megraud, F. (2006). Arcobacter butzleri: underestimated enteropathogen. Emerg. Infect. Dis. 12, 307-309. doi: 10.3201/eid1202.050570

Seemann, T. (2014). Prokka: rapid prokaryotic genome annotation. Bioinformatics 30, 2068-2069. doi: 10.1093/bioinformatics/btu153

Segata, N., Bornigen, D., Morgan, X. C., and Huttenhower, C. (2013). PhyloPhlAn is a new method for improved phylogenetic and taxonomic placement of microbes. Nat. Commun. 4:2304. doi: 10.1038/ncomms3304

Segata, N., Waldron, L., Ballarini, A., Narasimhan, V., Jousson, O., and Huttenhower, C. (2012). Metagenomic microbial community profiling using unique clade-specific marker genes. Nat. Methods 9, 811-814. doi: 10.1038/ nmeth.2066

Sundin, G. W., and Bender, C. L. (1996). Dissemination of the strA-strB streptomycin-resistance genes among commensal and pathogenic bacteria from humans, animals, and plants. Mol. Ecol. 5, 133-143. doi: 10.1111/j.1365-294X. 1996.tb00299.x

Toh, H., Sharma, V. K., Oshima, K., Kondo, S., Hattori, M., Ward, F. B., et al. (2011). Complete genome sequences of Arcobacter butzleri ED-1 and Arcobacter sp. strain L, both isolated from a microbial fuel cell. J. Bacteriol. 193, 6411-6412. doi: 10.1128/JB.06084-11

Ünver, A., Atabay, H., Sahin, M., and Celebi, Ö (2013). Antimicrobial susceptibilities of various Arcobacter species. Turk. J. Med. Sci. 43, 548-552. doi: 10.3906/sag-1207-115

Van den Abeele, A. M., Vogelaers, D., Van Hende, J., and Houf, K. (2014). Prevalence of Arcobacter species among humans, Belgium, 2008-2013. Emerg. Infect. Dis. 20, 1731-1734. doi: 10.3201/eid2010.140433

Van den Abeele, A. M., Vogelaers, D., Vanlaere, E., and Houf, K. (2016). Antimicrobial susceptibility testing of Arcobacter butzleri and Arcobacter cryaerophilus strains isolated from Belgian patients. J. Antimicrob. Chemother. 71, 1241-1244. doi: 10.1093/jac/dkv483

WHO (2018). Campylobacter Fact Sheet. Available at: http://www.who.int/ mediacentre/factsheets/fs255/en/

Wick, R. R., Schultz, M. B., Zobel, J., and Holt, K. E. (2015). Bandage: interactive visualization of de novo genome assemblies. Bioinformatics 31, 3350-3352. doi: 10.1093/bioinformatics/btv383

Wood, D. E., and Salzberg, S. L. (2014). Kraken: ultrafast metagenomic sequence classification using exact alignments. Genome Biol. 15:R46. doi: 10.1186/gb2014-15-3-r46

Yan, J. J., Ko, W. C., Huang, A. H., Chen, H. M., Jin, Y. T., and Wu, J. J. (2000). Arcobacter butzleri bacteremia in a patient with liver cirrhosis. J. Formos. Med. Assoc. 99, 166-169.

Yesilmen, S., Vural, A., Erkan, M. E., and Yildirim, I. H. (2014). Prevalence and antimicrobial susceptibility of Arcobacter species in cow milk, water buffalo milk and fresh village cheese. Int. J. Food Microbiol. 188, 11-14. doi: 10.1016/ j.ijfoodmicro.2014.07.006

Zankari, E., Hasman, H., Cosentino, S., Vestergaard, M., Rasmussen, S., Lund, O., et al. (2012). Identification of acquired antimicrobial resistance genes. J. Antimicrob. Chemother. 67, 2640-2644. doi: 10.1093/jac/dks261

Conflict of Interest Statement: The authors declare that the research was conducted in the absence of any commercial or financial relationships that could be construed as a potential conflict of interest.

Copyright (c) 2018 Hänel, Hotzel, Tomaso and Busch. This is an open-access article distributed under the terms of the Creative Commons Attribution License (CC BY). The use, distribution or reproduction in other forums is permitted, provided the original author(s) and the copyright owner(s) are credited and that the original publication in this journal is cited, in accordance with accepted academic practice. No use, distribution or reproduction is permitted which does not comply with these terms. 